\title{
Wireless Multimedia Sensor Networks based Quality of Service Sentient Routing Protocols: A Survey
}

\author{
Ronald Chiwariro $^{1}$ \\ Research Scholar, Computer Science Engineering \\ JAIN (Deemed-to-be University) \\ Bangalore, India
}

\author{
Dr. Thangadurai. $\mathrm{N}^{2}$ \\ Professor and Research Coordinator \\ Department of Electronics and Communication Engineering \\ JAIN (Deemed-to-be University), Bangalore, India
}

\begin{abstract}
Improvements in nanotechnology have introduced contemporary sensory devices that are capable of gathering multimedia data in form of images, audio and video. Wireless multimedia sensor networks are designed to handle such type of heterogeneous traffic. The ability to handle scalar and non-scalar data has led to the development of various real-time applications such as security surveillance, traffic monitoring and health systems. Since, these networks are an emergent of wireless sensor networks; they inherit constraints that exist in these traditional networks. Particularly, these networks suffer from quality of service and energy efficiency due to the nature of traffic. This paper presents the characteristics and requirements of wireless multimedia sensor networks and approaches to mitigate existing challenges. Furthermore, a review of recent research on multipath routing protocols and multi-channel media access protocols that have quality of service assurances and energy efficiency in handling multimedia data have included.
\end{abstract}

Keywords-Quality of service; multipath routing; multichannel media access control; energy efficiency

\section{INTRODUCTION}

Wireless Multimedia Sensor Networks (WMSNs) have enhanced the data gathering capability of the traditional Wireless Sensor Networks (WSNs) which were restricted only to gathering scalar data. WMSNs have sensor nodes equipped with cameras and microphones that enable these networks to gather multimedia data in various forms like live data streams, videos, audio, images and so on[1]. Recent advances in feature engineering, image-processing techniques, machine learning and communication technologies have given birth to various research to applications of WMSNs. Applications include health care industry, military and general surveillance systems, real time intelligent transportation systems and environmental monitoring [2], [3].

The WMSNs are, descendent of WSNs hence the same benefits such as self-organization, flexibility, disposition simplicity and scalability are also characteristic. However, the added features and capabilities in WMSNs present a number of challenges that are inherent with these constrained networks such as limited energy, storage, communication bandwidth as well as processing capacity. The large volumes of data generated by these multimedia networks to be reliably transmitted over the wireless medium in real-time further exacerbate these challenges. Research, on this domain, aims at the development of computation algorithms and protocols that are highly energy-efficient and Quality of Service (QoS) aware. Due to these variations, the solutions developed for WSNs cannot be directly applied to WMSNs. Therefore there is need to modify these techniques before they can be applied to WMSNs. Furthermore, new techniques at all layers from physical layer to application layer suitable for these networks are required. Surveys on such research ranging from hardware to the network model layers and other cross-layer designs are[1], [4]-[6]. Some extensive studies on various hardware and software architecture test beds are in [7]. Transport protocols designed to be reliable are in [8]. A comparison of energy efficient and QoS aware routing protocols is done in [9]-[11]. Accordingly, a review of QoS cognizant and multichannel Media Access Control (MAC) protocols are in [12], [13]. AlSkaif et al. present a comparative study on WSNs MAC protocols investigating their suitability on WMSNs through the analysis of some network parameters on node energy drain [14]. Authors in [15], [16] identifies cross-layer optimization solutions to problems inherent in WMSNs packet delivery, energy preservation and error recovery. Discussions of security requirements in WMSNs and classification of the security threats as well as some protection mechanisms are in [22], [23]. Finally,[19] discusses energy-efficiency issues with regard to all sensor application designs as well as extension of network lifetime while [20] [21] proposes a classification of energy-efficient target tracking schemes according to sensing and communication subsystems on a particular node.

This survey will thus concentrate on the important aspects required to deliver QoS-aware routing protocols in WMSNs, thus energy-efficiency, real-time multimedia streaming and data volumes. The paper will also highlight challenges and proffered solutions to guide related research. Network designers and architects will also immensely benefit from the clarity on characteristics and requirements of WMSNs as well as existing solutions. Furthermore, presented is a survey of communication MAC and routing protocols with emphasis on energy-efficiency, scalability, QoS guarantee, prioritization schemes, multipath routing and service differentiation [17][18]. The conclusion will also give future directions on discussed issues.

The remaining paper is as follows: Section 2 highlights the characteristics and design requirements of WMSNs with design challenges and existing remedies. A study of multipath routing protocols is done in Section 3 followed by WMSNs proposed MAC protocols. Lastly, Section 5 presents conclusions to the survey. 


\section{WiRELESS MULtimEdia SENSOR NETWORKS}

WMSNs are an emergent technology out of the traditional WSNs. As such, they inherit many constrains that exist in these networks as well as new challenges and requirements that come because of the requirement for real-time multimedia services and handling of increased volumes of data. The gathered data traffic handled by these networks requires delivery in real-time due to the nature of applications that require the data. Examples of such applications include security surveillance, health systems and traffic management systems. The multimedia data collected by the camera sensors is voluminous for a particular event; hence, bandwidth requirements for the transmissions are increased. As summarized in Table I, WMSNs have opened many doors to research due to their characteristics and capabilities. This section discusses the characteristics, design requirements of WMSNs as well as proposed approaches [24].

\section{A. Power Constraints}

The camera sensor nodes in WMSNs are generally batterypowered. The batteries are expected to power the sensor nodes for protracted periods without replacement. Therefore, the functionality of such nodes should take into cognizance these power constraints and limit energy consumption in its computations and communication. In traditional WSNs, energy drain due to computations can be insignificant compared to WMSNs where computations tend to consume extremely high energy. To, capture and processing of a simple frame in a vehicle tracking system can constitute up to $12 \%$ of total energy consumption of the overall event. It is therefore recommended to adopt energy-efficient algorithms in image processing [21] and likewise in video compression. Due to the large volumes of multimedia data to be transmitted, it is prudent that the communication protocols at every layer be energy-efficient. For example, the transport layer protocols reduce the number of control messages according to desired levels of reliability, with routing protocols employing load balancing and energy estimation techniques across the network and at the MAC layer protocols can avoid idle listening by inactive nodes. Dynamic power management is another important technique to be used as it ensures that idle components of a sensor node are selectively shutdown or hibernated to prevent unnecessary power consumption.

\section{B. Real-Time Multimedia Data}

In most applications involving multimedia data, QoS is difficult to achieve. Transmission of data to the sink without any packet loss or delays above a threshold is very crucial in WMSNs. Therefore there is a need to impose severe QoS demands on the networks. Delays cannot be tolerated in applications that involve multimedia data for example in security surveillance or traffic management systems. This implies that prioritization and service differentiation will play a pivotal role in these real-time systems. MAC protocols should give access or assign greater quality channels to higher priority data. Routing protocols need to select paths that will have the least delay to meet the required QoS as illustrated in. Reliability is also crucial in ensuring QoS to WMSNs. Retransmissions are done at transport layer for example in TCP while redundancy is at bit-level or at packet-level as presented in [8]. However, these methods must be used with consideration that they increase traffic hence consume more networks resources. The heterogeneous traffic in WMSNs that include multimedia and scalar data intended for different applications with varying QoS demands will require variable levels of priority even within the same traffic type.

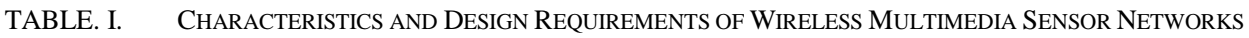

\begin{tabular}{|c|c|c|}
\hline Characteristics & Requirements & Design Approaches \\
\hline \multirow{2}{*}{ Power Constraints } & \multirow{2}{*}{ Energy efficiency } & $\begin{array}{l}\text { Energy-efficient computations } \\
\text { - } \quad \text { Image compression algorithms } \\
\text { - Video compression algorithms } \\
\text { Dynamic power management }\end{array}$ \\
\hline & & \begin{tabular}{ll}
\multicolumn{2}{l}{ Energy-efficient computations } \\
- & Transport Protocols \\
$-\quad$ & Routing protocols \\
$-\quad$ & MAC protocols \\
\end{tabular} \\
\hline \multirow[t]{2}{*}{ Real-time multimedia data } & \multirow[t]{2}{*}{ Quality of Service } & $\begin{array}{l}\text { Delay } \\
-\quad \text { Routing protocols } \\
\text { - } \quad \text { MAC protocols } \\
\text { Reliability } \\
-\quad \text { Routing protocols }[8] \\
-\quad \text { MAC protocols } \\
\end{array}$ \\
\hline & & $\begin{array}{l}\text { Prioritization and service differentiation } \\
-\quad \text { Routing protocols } \\
\text { - MAC protocols } \\
\text { Local processing }\end{array}$ \\
\hline \multirow{3}{*}{ Volumes of multimedia data } & \multirow[t]{2}{*}{ Reduction of data redundancy } & $\begin{array}{l}\text { Multimedia in-network processing } \\
\text { - Multimedia data fusion } \\
\text { - Multi-view video summarization } \\
\text { Distributed source coding }\end{array}$ \\
\hline & & In-network data storage and query processing \\
\hline & Higher Bandwidth Requirement & $\begin{array}{l}\text { Multipath routing } \\
\text { Multi-channel MAC protocols } \\
\text { Ultra Wideband technique }\end{array}$ \\
\hline
\end{tabular}




\section{Volumes of Multimedia Data}

Typically, WMSNs have limited bandwidth hence transmission of large volumes of sensory data presents a major challenge to QoS guarantee. Techniques for data compression and redundancy reduction are vital to decrease data volumes prior to transmission. One such technique is local processing where on-board analysis of the captured images is used to extract only important events. The downside of local processing is the requirement for added hardware resources. Another technique is In-network processing of multimedia data that encompass data fusion where the sink node collects heterogeneous data from various nodes and create a summarized version of events to reduce data redundancy and enhance inferences. To deal with the resource limitation problems associated with centrally coding data from multiple sensor cameras, WMSNs use distributed source coding (DSC) where encoding of data is done independently at each sensor before transmission to the sink for decoding. This reduces the power consumption as well as required hardware resources. Typically, WSNs transmit all collected data to the sink for subsequent processing and querying. Due to technological advancements, it is now possible to equip sensors with processors and flash memory that enable them to process and store data. After processing, only analyzed data is transmitted to the sink. In terms of queries, only the result is sent to the network after querying historical data. However, proper data ageing schemes needs to be incorporated into the local databases as they fill up in order to maintain data integrity. It is also important to note that the sensors will form distributed databases which require efficient query engines to retrieve the data efficiently. Mitigating the bandwidth constraint that is extreme in WMSNs due to the large volumes and nature of traffic is also an important factor in achieving QoS communications. At the MAC layer, sensor nodes can communicate simultaneously using different channels. Data traffic can be routed through multiple paths. However, radio equipment that has considerable bandwidth such as ultrawideband (UWB) can be utilized in WMSNs.

\section{Quality OF SERVICE Aware Multipath Routing PROTOCOLS FOR WMSNS}

Routing techniques for WSNs have been extensively studied over the years to improve communications. However, these techniques cannot be directly implemented in WMSNs due to variations with traditional WSNs. Routing in WSNs aims at finding the shortest path for transmission scalar data. Applying the same routing concepts to large volumes of multimedia data will result in network congestions and increased power drain on nodes. Therefore, the robust approach will be to send data in parallel through multiple paths. Routing in WSNs is particularly concerned with energy-efficiency whilst WMSNs also consider the QoS due to real-time traffic and reliability concerns.

This section presents some multipath routing protocols in WMSNs with QoS assurances. This survey looks at different protocols than those recently surveyed in. Furthermore, the chosen multipath routing protocols have single path routing support. For further comparison of the surveyed multipath routing protocols with QoS assurances, particularly to WMSNs refer to Table II.

TABLE. II. COMPARISON OF MULTIPATH ROUTING PROTOCOLS UNDER REVIEW

\begin{tabular}{|c|c|c|c|c|c|}
\hline Protocol & DGR & AntSensNet & Z-MHTR & GEAM & LCMR \\
\hline Routing Method & geographic routing & ant colony based routing & $\begin{array}{l}\text { ZigBee cluster tree } \\
\text { routing }\end{array}$ & $\begin{array}{l}\text { geographic } \\
\text { routing }\end{array}$ & $\begin{array}{l}\text { ad-hoc on-demand distance } \\
\text { vector routing }\end{array}$ \\
\hline Routing Metric & $\begin{array}{l}\text { geographic distance } \\
\text { and deviation angle }\end{array}$ & $\begin{array}{l}\text { pheromone value of residual } \\
\text { energy, delay, packet loss rate } \\
\text { and available memory }\end{array}$ & network address & $\begin{array}{l}\text { geographic } \\
\text { distance }\end{array}$ & end-to-end delay \\
\hline Routing States & $\begin{array}{l}\text { one hop neighbour } \\
\text { table }\end{array}$ & $\begin{array}{l}\text { one hop neighbour table, } \\
\text { routing pheromone table }\end{array}$ & $\begin{array}{l}\text { one hop neighbour } \\
\text { table, tree branches } \\
\text { used in routing and/ } \\
\text { or interfering node } \\
\text { table }\end{array}$ & $\begin{array}{l}\text { one hop neighbour } \\
\text { table, district } \\
\text { information }\end{array}$ & routing table \\
\hline Disjoint Paths & yes & yes & yes & yes & no \\
\hline QoS Metrics & $\begin{array}{l}\text { reliability and } \\
\text { throughput }\end{array}$ & reliability, delay, throughput & throughput & throughput & delay \\
\hline Path Recovery & yes & yes & no & yes & no \\
\hline Scalability & good & good & good & good & poor \\
\hline Congestion Control & no & yes & no & no & no \\
\hline Prioritization & no & yes & no & no & no \\
\hline Service Differentiation & no & yes & no & no & no \\
\hline Energy Efficiency & medium & medium & good & good & poor \\
\hline Clustered & no & yes & yes & no & no \\
\hline Interference Aware & no & no & yes & yes & no \\
\hline Year & 2007 & 2010 & 2014 & 2013 & 2017 \\
\hline
\end{tabular}


A multipath routing protocol based on ant colony optimization called AntSensNet with QoS assurances is presented in. It has three phases of operation: Formation of the cluster, route discovery phase, data transmission and route maintenance. The cluster formation is initiated by the sink that releases some cluster ants (CANTs). Those within close proximity to the sink are selected as cluster heads $(\mathrm{CH})$ and will receive the CAs first. Upon receiving the CANTs, they will be responsible for the reduction of the time-to-live (TTL). The cluster head will then advertise the CANTs to non-cluster heads within its communication radius so that those who are willing to join the cluster can join. Once clusters are formed, the $\mathrm{CH}$ begins route discovery. Each $\mathrm{CH}$ manages a pheromone table and shares with its neighbors according to traffic classes following four parameters i.e. Energy, packet drop, memory and delay. Traffic specific paths to the sink is created by broadcasting a forward ant (FANT) which will collect traversed node identities and the four parameters (queue delay, ratio of packet, residual energy and available memory) as it propagates. When a node receives a FANT, an update is done to its information before sending it to the next hope that satisfies the QoS requirements and a corresponding backward ant (BANT) is transmitted in the reverse path for path reservation. On receipt of the BANT, nodes update their pheromone tables. For establishment of multiple paths for video transmission, a video forward ant (VFANT) is broadcasted in the same manner as the FANT and the sink responds by sending multiple VBANTs. The VBANTs will be used to choose paths for sending video data. Once routes are ready data, delivery starts. A maintenance ant (MANT) is used for route maintenance. This protocol gives differentiated service to ensure QoS delivery by offering each traffic separate routes. The use of cluster heads is a drawback on scalability. However, the multipath routing technique is viable for video data only.

Z. Bidai et al. proposed the ZigBee Multipath Hierarchical Tree Routing (Z-MHTR) protocol. It allows source to use nonparent neighbors to search for other paths. The source node maintains a record of all branches used for tree routing (TR). The source node will construct disjoint paths using three basic principles. If a selected next hop node branch has not been utilized for TR path by the source node then a node disjoint can be established from that node to the sink using TR. If the branch has already been utilized for TR path by the source then the next hop will depend upon the depth of a node common to the TR path used by the source and the node that has used the node branch for TR. If all neighbor's branches have been utilized in TR then it selects the neighbor node that is not in any TR path. The rules are applied to any subsequent nodes until the sink. The number of disjoint paths corresponds to the number of branches forming the topology. Furthermore, the author proposed for reduction of interference in which nodes lists interfering neighbors except the ones on the same paths. This is done by checking whether they can hear data packets that are not destined to them. The disjoint paths that reduce inter-path interloping are preferred. Based on the ZigBee tree topology and address assignment, multipath routing is achieved through neighbor table and a record of routing tree usage on a particular branch. The further work mitigates multiple paths interferences caused by route coupling. However, the restriction is only to ZigBee tree topology hence the paths are proportionate to available branches.

M. Chen et al. recommended the directional geographical routing (DGR) protocol for real-time video communications. The nodes in this protocol implement the global coordinate system to create virtual coordinates upon receipt of a broadcast probe. The virtual coordinates are obtained by mapping the source and sink position along the $\mathrm{x}$ axis to the destination or intermediate node. A node is selected to be a forwarding candidate only if it falls within the transmission range, the optimal mapping location and the threshold of the source. Next hop will be a candidate that has the smallest distance to the optimal mapping hence; it will have a smaller timer than other competing nodes. If a timer expires, the node sends a reply message REP to the source. On receipt of an REP, the source confirms with SEL message. Nodes that hear the REP or SEL cancels their timers. The winner node will not establish any other path to the same source in order to guarantee path disjointedness. In turn, the connected node will send its own probing messages following the same procedure with an adjusted deviation angle to create a path towards the sink. For establishment of multiple paths, the source will send a number of probe messages with variations in the initial deviation angle. For video routing, the source broadcasts the complete frame initially to all single hop neighbors. Those neighbors within the chosen paths will retransmit the video using respective paths only those packets specified by the source. The packet delivery in this protocol is fast and reliable through multipath and the forwarding equivalence class. It also scales well due to the stateless geographic based routing paradigm. However, if a node fails, the path recovery takes longer as well as the new route discovery. In addition, it considers only a single active source for video transmissions that might not be practical in some scenarios.

A. Bhattacharya and K. Sinha following the principles of ad-hoc on-demand distance vector routing (AODV) developed the least common multiple routing (LCMR) protocol. As opposed to calculating the shortest path by number of hops, it uses the routing time taken or end-to-end delay to choose multiple paths. During route discovery, the route reply message RREP has to arrive before the deadline otherwise it will not be accepted. The source node uses the RREP message to check the routing time taken by the corresponding route request message RREQ before reaching the destination. From the accepted $x$ paths that have routing time $\left\{T_{1}, T_{2}, \ldots, T_{x}\right\}$, it calculates the least common multiple $\mathrm{L}$ of $\left\{T_{1}, T_{2}, \ldots, T_{x}\right\}$. The packets sent over path $i$ are decided such that $=\sum_{i=1}^{x} L / T i$ packets, $\mathrm{L} / \mathrm{T}_{i}$ packets will be routed along that path $i$. The total time it takes to deliver $k$ packets gives the maximum routing time $\mathrm{T}_{\max }$ of $\left\{T_{1}, T_{2, \ldots}, T_{x}\right\}$. This protocol ensures avoidance of congested routes through the end-to-end calculation of routing time during its route discovery process. In order to reduce the transmission time, the number of packets allotted to a particular route is reduced according to time $\mathrm{L}$ and the routing time $\mathrm{T}_{i}$ of the path. However, this may lead to early node death if most traffic is continuously routed through a node with least end-to-end routing time. Adaption to congestion and route breakage needs improvement. 
Unlike DGR, that uses the deviation angle for controlling the directions of multiple paths, $\mathrm{Li}$ et al proposed the division of the topology into different districts for specific paths using the geographic energy-aware non-interfering multipath routing (GEAM) protocol. After division into virtual coordinates just like in DGR, the source and sink areas are restricted within the transmission radius. Each packet is piggybacked with boundary information of the selected district by the source before transmission. The subsequent nodes will then use greedy perimeter stateless routing (GPSR) to forward the packet to the respective district. For load balancing and even distribution of energy, GEAM the data transmissions are organized in runs of same lengths. To further avoid interference within multiple routing paths, it applies division of runs into three rounds, where a district $\mathrm{D}_{x}$ belongs to round $k$ if $\mathrm{D}_{x} \% 3=k$. During the first run, loads are distributed evenly to all districts. After each run, the sink collects residual energy from all nodes within a district and sends back to the source. Based on these statistics the source adjusts the rate of utilization for every district and those with higher energy levels get more loads in the next run. GEAM achieves balanced traffic loads and energy consumption as well as avoids interference by the division to the topology into various districts. Scalability is also guaranteed using GPSR. However, piggybacking every packet with border information and making it collect network statistics increases the overhead. It also does not consider some QoS metrics such as delay and reliability that are of paramount importance to delivery of multimedia data.

\section{Quality of Service Aware Media ACCESS CONTROL PROTOCOLS FOR WMSNS}

MAC protocols present a challenge during their design and implementation when aiming for energy efficiency and coordinating transmission of large volumes of multimedia sensory data and meeting QoS in MWSNs. The dynamic and burst traffic predominant in WMSNs it requires application of duty cycling techniques in saving energy deeper analysis. Reduction of collisions is also an important factor in MAC protocol design especially when it involves real-time multimedia data. Controlling media access through prioritization and differentiation of services is also an important factor when handling heterogeneous traffic. This section will elaborate some of the energy-efficient MAC protocols that have QoS assurances. A summary of the same is in Table III.

M. Arifuzzaman et al. proposed the intelligent hybrid MAC (IH-MAC) protocol. This protocol combines CSMA/CA and TDMA techniques as a single mechanism that implements local synchronization. The protocol prioritizes the node holding data with high QoS such as real-time data. If nodes have same priority and mapped to same slot, then they contend for that slot. For energy preservation, it adjusts its transmission output during the contentions. The protocol scales well and reduces collisions as well as improves on channel utilization and access delays that are challenges in CSMA/CA by fusion of CSMA/CA and TDMA.

TABLE. III. COMPARISON OF MEDIA ACCESS CONTROL PROTOCOLS

\begin{tabular}{|c|c|c|c|c|c|c|c|}
\hline Protocol & EQ-MAC & Saxena & Diff-MAC & MQ-MAC & IH-MAC & AMPH & PA-MAC \\
\hline MAC Mechanism & $\begin{array}{l}\text { hybrid of } \\
\text { CSMA/ CA } \\
\text { and TDMA }\end{array}$ & CSMA/ CA & CSMA/ CA & IEEE 802.15.4 & $\begin{array}{l}\text { hybrid of } \\
\text { CSMA/ CA and } \\
\text { TDMA }\end{array}$ & $\begin{array}{l}\text { hybrid of } \\
\text { CSMA/ CA and } \\
\text { TDMA }\end{array}$ & IEEE 802.15 .4 \\
\hline Synchronization & $\begin{array}{l}\text { global, } \\
\text { precise }\end{array}$ & not required & not required & local, precise & local, precise & global, precise & global, precise \\
\hline QoS Guarantee & delay & $\begin{array}{l}\text { throughput, } \\
\text { delay }\end{array}$ & reliability, delay & reliability, delay & delay & reliability, delay & throughput, delay \\
\hline $\begin{array}{l}\text { Prioritization } \\
\text { Scheme }\end{array}$ & traffic types & traffic types & $\begin{array}{l}\text { traffic types, } \\
\text { traversed hop } \\
\text { count of packets }\end{array}$ & $\begin{array}{l}\text { traffic types, } \\
\text { packet lifetime }\end{array}$ & traffic types & $\begin{array}{l}\text { traffic types, } \\
\text { dynamic }\end{array}$ & traffic types \\
\hline $\begin{array}{l}\text { Service } \\
\text { Differentiation } \\
\text { Scheme }\end{array}$ & $\begin{array}{l}\text { dynamic slot } \\
\text { allocation }\end{array}$ & $\begin{array}{l}\text { adaptive } \\
\text { contention } \\
\text { window, } \\
\text { dynamic duty } \\
\text { cycle }\end{array}$ & $\begin{array}{l}\text { adaptive } \\
\text { contention } \\
\text { window, } \\
\text { dynamic duty } \\
\text { cycle, weighted } \\
\text { fair queueing }\end{array}$ & $\begin{array}{l}\text { dynamic channel } \\
\text { allocation, } \\
\text { dynamic slot } \\
\text { allocation, } \\
\text { adaptive } \\
\text { contention } \\
\text { window } \\
\end{array}$ & $\begin{array}{l}\text { adaptive } \\
\text { contention } \\
\text { window, } \\
\text { dynamic slot } \\
\text { allocation }\end{array}$ & $\begin{array}{l}\text { adaptive } \\
\text { contention } \\
\text { window, } \\
\text { dynamic slot } \\
\text { allocation }\end{array}$ & $\begin{array}{l}\text { dynamic channel } \\
\text { access control }\end{array}$ \\
\hline Scalability & poor & good & good & medium & medium & poor & poor \\
\hline $\begin{array}{l}\text { Adaptation to } \\
\text { Dynamic Traffic }\end{array}$ & good & medium & medium & poor & good & good & poor \\
\hline Collision Rate & low & medium & medium & low & low & low & high \\
\hline Energy Efficiency & good & medium & medium & good & medium & poor & good \\
\hline Message Passing & no & no & yes & no & no & yes & no \\
\hline Clustered & yes & no & no & yes & yes & no & no \\
\hline Year & 2008 & 2008 & 2011 & 2015 & 2013 & 2014 & 2016 \\
\hline
\end{tabular}


An energy-efficient hybrid MAC scheme (EQ-MAC), was proposed by Yahya and Ben-Othman. It uses the cluster mechanism in which the cluster head schedules slots using TDMA. It uses frames for communication. The cluster head sends the initial broadcast frame for synchronization. Once synchronization is completed, the cluster members start transmission of data through the cluster head. The cluster head issues TDMA slots upon request from the cluster members with consideration of traffic priorities. The cluster head then broadcasts allocated TDMA slots to cluster members for transmissions to begin. Sleep mechanism will also apply to those cluster members without data to transmit. Real-time data is placed in a queue that is served instantaneously. The sleep mechanism saves energy and channel utilization. The protocol assures delivery of real-time data especially multimedia due to prioritization of traffic. However, this may starve low priority traffic.

An efficient QoS provisioning protocol by M. Souil (AMPH), is a hybrid channel access method. The notable difference between AMPH and IH-MAC is that the latter is CSMA/CA centered and AMPH is TDMA centered. AMPH divides transmissions into slots and two-hop radius for each node. Prioritization for medium access is done by separation of real-time and best effort traffic and based on slot ownership. Contending nodes are separated into four groups according to traffic priority: real-time by owner, real-time by non-owner, best effort by owner and best effort by non-owner. To avoid starvation, the protocol allows best effort traffic ahead of real-time traffic in limited slots per cycle. To conserve energy, it allows nodes to switch of their radios in the waiting state. The use of any slot coupled with traffic prioritization achieves optimum channel utilization and QoS guarantees to heterogeneous traffic. However, there is need for a robust differentiation of traffic that caters for more traffic types that exist in WMSNs.

Bhandari et al. proposed a multi-channel priority based adaptive MAC protocol (PA-MAC) that is based on the IEEE 802.15.4 standard. The protocol traffic classification is grouped into four categories according to priority: emergency (medical), on-demand, normal, non-medical. It uses the contention access periods (CAP) for the four classifications of traffic. Traffic with higher priority is allowed access to slots for lower priority traffic and the lower priority traffic transmits during the contention free period (CFP). The nodes enter into sleep until next transmission. Collisions are mitigated by traffic differentiation and transmission of lower priority data (e.g. multimedia data in medical scenario) at CFP. However, the protocol gives less priority to multimedia data hence it cannot be applied directly to WMSNs.

Related CSMA/ CA based protocols with QoS assurances were proposed by Saxena et al. and Diff-MAC. The protocols use adaptive contention window $(\mathrm{CW})$ and dynamic duty cycling mechanisms. The $\mathrm{CW}$ sizes for real-time traffic are set to be less than low priority traffic. The protocols differ in that, Saxena et al. aims for fairness by making sensors adjust their CW size after checking with neighboring sensors if chances of a collision remain after last $\mathrm{CW}$ size changes whereas sensors in Diff-MAC continue to change their CW sizes towards the threshold CW size. Diff-MAC also employs the hybrid weighted fair queuing (WFQ) technique to allow channel access to real-time traffic while Saxena et al. uses a FIFO mechanism. Diff-MAC avoids starvation to same traffic type by prioritization of packets belonging to the same queue prioritizing them based on traversed hops. It further segments video frames and transmit the in bursts to lower retransmission cost. Both protocols use the dynamic duty cycle technique. The protocols offer good QoS, fairness and energy-efficiency in WMSNs. However, constantly monitoring of various states in a network leads to idle listening and as for Diff-MAC, the constant intra-queue prioritizations may not scale well with high traffic.

MQ-MAC is a cluster based slotted CSMA/ CA MAC protocol. The cluster head is responsible for key responsibilities that include channel sensing, time slot allotments and channel allocation. It divides its super frame into active and sleep periods, with the active being subdivided into three phases namely; sensing, channel selection and data transmission requests. Once the cluster head receives results of channel sensing and transmission requests from the cluster members, it will allocate slots and transmission channels. QoS is guaranteed through slot allocation. The requests once received from cluster members are classified according to arrival time and traffic type as well as consideration of the packet lifetime. Early slots are allocated to requests with higher priority. The slot allocations are allows data traffic from cluster members to the cluster head to be collision free. After the transmission phase, the sensor nodes will sleep and wake up when another super frame starts. QoS is guaranteed through allocation of slots and channels for different traffic types according to priority. However, the presence of many control messages during sensing and switching are not desirable due to overheads.

\section{CONCLUSION}

WMSNs are becoming more popular in various IoT applications due to their ability to handle heterogeneous data from various sensory devices. Considerable research has been done to enhance these networks. However, some challenges are still prevalent due to the distinctive characteristics of the WMSN and resource constraints. This paper covered the unique characteristics and requirements for WMSNs as well as some design approaches to the constraints highlighted. Furthermore, the survey includes multipath routing protocols and MAC protocols, which are two important communication parameters to improve QoS provision in any network. Multipath routing is significant to the provision of QoS and delivery of multimedia data in WMSNs. These protocols are able to distribute the voluminous multimedia data across the network, thus balancing the load as well as energy consumption. It is important for the protocols to counter interference in multiple parallel paths to avoid route coupling issues. However, most multipath routing protocols consider load balancing and energy management without due diligence for other QoS metrics such as prioritization, and differentiation of service. Traffic in these networks is heterogeneous in nature therefore prioritization and service differentiation should not only be fixed to a particular type of traffic as in the case with most protocols that dedicate only to video traffic. Route recovery and congestion control must also 
be given great significance to improve QoS in WMSNs. Finally, Efficient MAC protocols intended for WMSNs must be able to handle heterogeneous traffic and vast volumes of multimedia data. In literature, there exist CSMA/ CA based MAC protocols that are scalable and adapt to different variable traffic situations although suffer bottlenecks in QoS provision and energy efficiency. Hybrid protocols combining CSMA/ CA and TDMA are promising to be an important part of WMSNs since CSMA/ CA and TDMA are used to handle low data rates and high data rates respectively thereby improving throughput and the reduction of collisions. It is important to note that QoS is of great importance to WMSNs hence the future research focus should be on handling multimedia data collected by camera sensors.

\section{REFERENCES}

[1] T. Almalkawi, M. G. Zapata, J. N. al-Karaki, and J. Morillo-Pozo, "Wireless multimedia sensor networks: Current trends and future directions," Sensors, vol. 10, no. 7, pp. 6662-6717, 2010.

[2] T. Semertzidis, K. Dimitropoulos, A. Koutsia, and N. Grammalidis, "Video sensor network for real-time traffic monitoring and surveillance," IET Intell. Transp. Syst., vol. 4, no. 2, p. 103, 2010.

[3] N. B. Bo et al., "Human mobility monitoring in very low resolution visual sensor network," Sensors (Switzerland), vol. 14, no. 11, pp. 20800-20824, 2014.

[4] S. Soro and W. Heinzelman, "A Survey of Visual Sensor Networks," Adv. Multimed., vol. 2009, pp. 1-21, 2009.

[5] A. Sharif, V. Potdar, and E. Chang, "Wireless multimedia sensor network technology: A survey," IEEE Int. Conf. Ind. Informatics, no. May 2014, pp. 606-613, 2009.

[6] I. F. Akyildiz, T. Melodia, and K. R. Chowdury, "Wireless multimedia sensor networks: A survey," IEEE Wirel. Commun., vol. 14, no. 6, pp. 32-39, 2007.

[7] I. F. Akyildiz, T. Melodia, and K. R. Chowdhury, "Wireless multimedia sensor networks: applications and testbeds," Proc. IEEE, vol. 96, no. 10, pp. $1588-1605,2008$.

[8] M. A. Mahmood, W. K. G. Seah, and I. Welch, "Reliability in wireless sensor networks: A survey and challenges ahead," Comput. Networks, vol. 79, pp. 166-187, 2015.

[9] M. Radi, B. Dezfouli, K. A. Bakar, and M. Lee, "Multipath routing in wireless sensor networks: Survey and research challenges," Sensors, vol. 12, no. 1, pp. 650-685, 2012.

[10] A. M. Zungeru, L.-M. Ang, and K. P. Seng, "Classical and swarm intelligence based routing protocols for wireless sensor networks: A survey and comparison," J. Netw. Comput. Appl., vol. 35, no. 5, pp. 1508-1536, Sep. 2012.
[11] S. Ehsan and B. Hamdaoui, "A Survey on Energy-Efficient Routing Techniques with QoS Assurances for Wireless Multimedia Sensor Networks," IEEE Commun. Surv. Tutorials, vol. 14, no. 2, pp. 265-278, 2012.

[12] O. D. Incel, "A survey on multi-channel communication in wireless sensor networks," Comput. Networks, vol. 55, no. 13, pp. 3081-3099, Sep. 2011.

[13] M. A. Yigitel, O. D. Incel, and C. Ersoy, "QoS-aware MAC protocols for wireless sensor networks: A survey," Comput. Networks, vol. 55, no. 8, pp. 1982-2004, 2011.

[14] T. AlSkaif, B. Bellalta, M. G. Zapata, and J. M. BarceloOrdinas, "Energy efficiency of MAC protocols in low data rate wireless multimedia sensor networks: A comparative study," Ad Hoc Networks, vol. 56, pp. 141-157, Mar. 2017.

[15] D. G. Costa and L. A. Guedes, "A survey on multimedia-based crosslayer optimization in visual sensor networks," Sensors, vol. 11, no. 5, pp. 5439-5468, 2011.

[16] N.Thangadurai, Dr.R.Dhanasekaran and R.D.Karthika, "Dynamic Energy Efficient Topology for Wireless Ad hoc Sensor Networks", WSEAS Transactions on Communications, Vol. 12, Iss. 12, pp. 651660, 2013.

[17] L. D. P. Mendes and J. J. P. C. Rodrigues, "A survey on cross-layer solutions for wireless sensor networks," J. Netw. Comput. Appl., vol. 34, no. 2, pp. 523-534, 2011.

[18] N. Thangadurai and Dr.R.Dhanasekaran, "Energy Efficient Cluster based Routing Protocol for Wireless Sensor Networks", International Journal of Computer Applications, Vol. 71, No. 7, pp. 43-48, 2013.

[19] N.Thangadurai, Dr.R.Dhanasekaran and R.D.Karthika, "Dynamic Traffic Energy Efficient Topology based Routing Protocol for Wireless Ad hoc Sensor Networks", International Review on Computers and Software, Vol. 8, No. 5, pp. 1141-1148, 2013.

[20] T. Winkler and B. Rinner, "Security and Privacy Protection in Visual Sensor Networks," ACM Comput. Surv., vol. 47, no. 1, pp. 1-42, 2014.

[21] M. Guerrero-Zapata, R. Zilan, J. M. Barceló-Ordinas, K. Bicakci, and B. Tavli, "The future of security in Wireless Multimedia Sensor Networks : A position paper," Telecommun. Syst., vol. 45, no. 1, pp. 77-91, 2010.

[22] T. Rault, A. Bouabdallah, and Y. Challal, "Energy efficiency in wireless sensor networks: A top-down survey," Comput. Networks, vol. 67, no. March, pp. 104-122, 2014.

[23] O. Demigha, W. K. Hidouci, and T. Ahmed, "On Energy efficiency in collaborative target tracking in wireless sensor network: A review," IEEE Commun. Surv. Tutorials, vol. 15, no. 3, pp. 1210-1222, 2013.

[24] S. Bhandari and S. Moh, "A priority-based adaptive MAC protocol for wireless body area networks," Sensors (Switzerland), vol. 16, no. 3, 2016. 